\title{
Efficacy of Cereal-based Oral Nutrition Supplement on Nutritional Status, Inflammatory Cytokine Secretion and Quality of Life in Cancer Patients Under Cancer Therapy
}

\author{
Jin-Min Kim1, Beom-Seok Song ${ }^{2}$, Sung-Gil Hong ${ }^{3}$, Hee-Jung Sohn ${ }^{4}$, Hyunwook Baik ${ }^{5}$, Mi-Kyung Sung ${ }^{1}$ \\ ${ }^{1}$ Department of Food and Nutrition, College of Human Ecology, Sookmyung Women's University, Seoul, ${ }^{2}$ Department of \\ Biotechnology, Advanced Radiation Technology Institute, Korea Atomic Energy Research Institute, Jeongeup, ${ }^{3}$ Erom Institute \\ of Life Sciences, Chuncheon, Departments of ${ }^{4} \mathrm{Hemato}-$ Oncology and ${ }^{5}$ Clinical Nutrition Medicine, Bundang Jesaeng Hospital, \\ Seongnam, Korea
}

\section{J Cancer Prev 2020;25(1):55-63 \\ https://doi.org/10.15430/JCP.2020.25.1.55}

The original version of this article contained errors in the name and affiliation of the Third author which was given incorrectly as Bum-Seok Song and Korea Atomic Energy Institute. The author's name and affiliation should have been written as BeomSeok Song and Korea Atomic Energy Research Institute. And, the order of the second and third authors was changed.

The authors would like to apologize for this mistake and any inconvenience it may have caused. 